\title{
SUCCESSFUL HIGH POWER TEST OF A PROTON LINAC BOOSTER (LIBO) PROTOTYPE FOR HADRONTHERAPY
}

\author{
B. Szeless, P. Berra, E. Rosso, M. Vretenar, CERN, Geneva, Switzerland \\ U. Amaldi, K.R. Crandall, D. Toet, M. Weiss, R. Zennaro, TERA Foundation, Novara, Italy \\ C. Cicardi, D. Giove*, C. De Martinis, University and INFN(*) Milan, Italy \\ D. Davino, M.R. Masullo*, V.G. Vaccaro, University and INFN(*) Naples, Italy
}

\begin{abstract}
The linac booster (LIBO) project aims to build a $3 \mathrm{GHz}$ proton linac to give the beam from $50-70 \mathrm{MeV}$ cyclotrons, which exist in several laboratories and hospitals, a final energy of $200 \mathrm{MeV}$. This will allow the treatment of deepseated tumours. A prototype of the first LIBO module was designed, constructed and RF tested by a collaboration of CERN, University and INFN of Milan, University and INFN of Naples, and the TERA Foundation. Low power RF measurements have shown good field uniformity and stability along the axis of the four tanks composing the LIBO module. In December 2000, full power RF measurements at a repetition rate of $100 \mathrm{~Hz}$ have been performed at CERN. After a very short conditioning period, an accelerating gradient approaching $30 \mathrm{MV} / \mathrm{m}$ has been easily achieved in the tanks, well above the nominal $15.8 \mathrm{MV} / \mathrm{m}$. The particularities of the design and the reasons for the successful performance are discussed.
\end{abstract}

\section{INTRODUCTION}

The LIBO is a $2.998 \mathrm{GHz}$ side-coupled linear accelerator [1], composed of nine 4-tank modules, of which the first one has been built as a prototype at CERN and successfully tested with full RF power in the LEP Injector Linac (LIL). The high power tests are described in the following section, while design, production and RF aspects, which contributed to the excellent behaviour of the prototype, are discussed in Sections 3 and 4.

\section{HIGH POWER TEST}

The LIBO module prototype, tuned and vacuum tested, was installed in the LIL area in November 2000, after the operation of LEP was over. It was connected to the vacuum equipment and thermostat-controlled water supply available in the LIL area, and powered by the klystron-modulator test system. Figure 1 shows the LIBO module installed at LIL: one distinguishes the waveguide of the RF feeder line as well as valves and tubes supplying the water to the channels of the cooling plates brazed on either side of each tank. The vacuum manifold with the ion and turbo pumps is placed underneath the LIBO. Fine tuning of the frequency during the power tests was done by changing the temperature of the LIBO structure. This was achieved by adjusting the temperature of the water circulating in the cooling plates [2].

The repetition rate during the tests was $100 \mathrm{~Hz}$ and the conditioning started with a pulse length of only $2 \mu \mathrm{s}$.

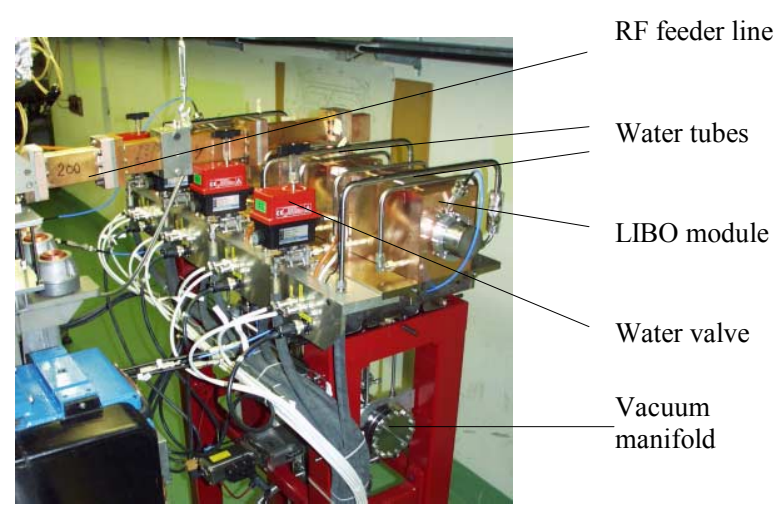

Figure 1: LIBO module installed in the LIL tunnel

After about 72 hours of conditioning, the nominal accelerating field level of $15.8 \mathrm{MV} / \mathrm{m}$ (peak power $\mathrm{P}=4.7$ MW) was exceeded, without multipactoring and with a very limited number of breakdowns. Having extended the pulse length to $5 \mu \mathrm{s}$, an accelerating field level of 27.5 $\mathrm{MV} / \mathrm{m}(\mathrm{P}=14.2 \mathrm{MW}$ ) was reached about 14 hours later (the corresponding computed maximum surface field was 2.6 times the Kilpatrick limit). A further increase of the $\mathrm{RF}$ input power was unfortunately prohibited by the power limit of the installed circulator. There were, however, no indications that the limit of the field level in LIBO had been reached. The field level in the module was obtained from the input power and the previously measured shunt impedance (see Section 4). Five pick-up loops placed at the input and output of LIBO and in the three bridge couplers allowed the monitoring of the field distribution in the prototype.

To get an independent confirmation of the high field level really present in the prototype, the structure temperature was measured as a function of injected power and of water flow. Thermocouples fixed on each tank, and platinum resistive probes mounted on water tubes provided a precise temperature measurement. By varying the water flow for one of the tanks, temperature gradients could be achieved with respect to the three others. The results obtained for different RF power levels were compared with computations: the agreement for the whole set of measurements was within $7 \%$.

A second check made use of an $\mathrm{NaI}(\mathrm{Tl})$ detector and measured the end point of the bremstrahlung X-ray spectra emitted from the cavities. The high level of background allowed only a rough estimate of the field level in the tanks, but agreed with the other measurement. 


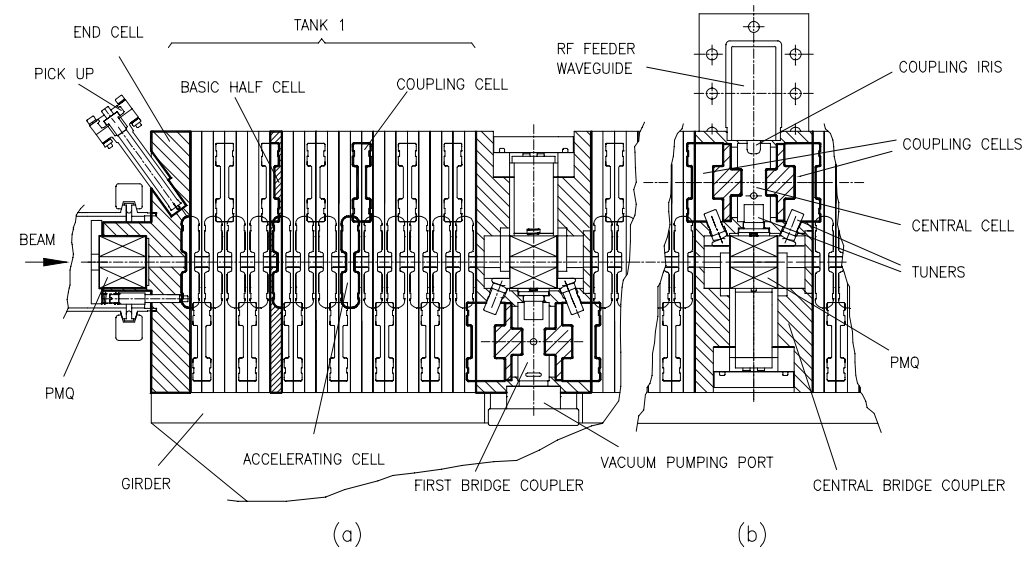

Figure 2: Partial drawing of the LIBO prototype. a) First tank with end cell and first 3-cell bridge coupler. b) Central 3-cell bridge coupler with RF feeder waveguide

\section{DESIGN AND PRODUCTION}

The prototype contains, as each LIBO module, four accelerating tanks, three bridge couplers and two end cell plates; see Figure 2. Five permanent magnet quadrupoles (PMQ) are placed in the two end cells and the three bridge couplers. The first and third bridge coupler have vacuum ports to be connected to a vacuum manifold, while the RF power is fed into the central bridge coupler via a matched waveguide.

The LIBO structure is composed of three types of elementary building blocks only: the basic half cell plates, the bridge couplers and the end cell plates.

The basic half cell plates, containing an accelerating half cell and a coupling half cell, are made of laminated OFE copper. They all have the same dimensions in a tank. Bridge couplers and end cells are of forged OFE copper, with brazed $316 \mathrm{LN}$ stainless steel flanges for vacuum openings and RF pick-ups, and fixation slugs.

All the elements have been machined on CNC lathes and milling machines. The pre-machined basic half cell plates were stress-relieved in air at $250^{\circ} \mathrm{C}$ to obtain, after final machining, the $20 \mu \mathrm{m}$ planarity required for vacuum brazing of the structure. Special attention has been paid to the precision of the coupling slot between the accelerating and coupling half cells. Its length, constant over the whole prototype, was precisely machined by milling to better than $0.1 \mathrm{~mm}$.

A thorough cleaning of all elements has been made prior to brazing, and a careful insertion of the brazing alloy as well as the stepwise assembly were done in a clean room under laminar air flow.

For the brazing, done in an all-metal vacuum oven, two methods have been applied: with wires in premachined grooves and with foils. In both, standard silver-based alloys have been used, at temperatures decreasing from $850^{\circ} \mathrm{C}$ to $750^{\circ} \mathrm{C}$, in accordance with the four brazing steps required. The components of the module were brazed in seven separate sub-assemblies during further manipulations.

(four tanks and three bridge couplers), which were then piled up vertically in the oven for the last two brazing steps. Figure 3 shows the LIBO module placed vertically in the oven; thus the brazing surfaces are horizontal, with brazing grooves facing down. All the lateral tuning rods in the accelerating and coupling cells, cut to the right length (see Chapter 4), were fixed in position and blocked with ceramic pieces and molybdenum springs.

After the last brazing step concerning the lateral cooling plates, the module was fixed on a rigid girder to guarantee stiffness

Leak detection after brazing was followed by a vacuum test of the fully equipped module.

Vacuum levels of $5 \times 10^{-7} \mathrm{mbar}$ and $5 \times 10^{-8} \mathrm{mbar}$ have

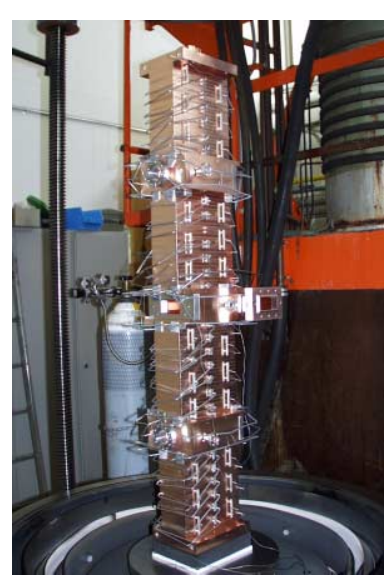

Figure 3: LIBO prototype in the oven for the penultimate brazing been reached in the module prototype and in the vacuum manifold, respectively. This is much better than the design requirement of $10^{-6}$ mbar.

After the final brazing, a 3D metrology measurement with an accuracy of $\pm 3 \mu \mathrm{m}$ has been performed. The results showed that the PMQ axis are within a circle of $0.1 \mathrm{~mm}$ in $\mathrm{x}, \mathrm{y}$, in accordance with the beam dynamics specifications.

\section{RF TUNING}

The frequency adjustment procedure was based on three tuning tools: tuning rings, tuning rods and screw tuners. The tuning rings and rods were available in each half cell, while the variable screw tuners equipped the end cells and the bridge couplers. Each tool was used at a given stage of the prototype production. The tuning ring allowed the machining tolerances of the inner cell surface to be relaxed. Before brazing the half-cell plates were measured individually and in partial ensembles (tanks); the results determined the remachining of the tuning rings.

The adjustment of the tuning rods was done before the vertical brazing of the module. For the tuning of the 3-cell magnetically coupled bridge couplers, neither tuning rods nor rings have been used. Once finished, the bridges have been tuned with adjustable screw 
tuners, a facility that could be used also after the final brazing (see Figure 2). The tuning procedure is based on the fact that, for a coupled resonator system, the only direct measurable quantities are the resonant frequencies of the whole structure, which differ from the single cell values. For a three-coupled-cell system like the 3-cell bridge, the unknown single cell frequencies are $\left(\omega_{1}, \omega_{2}, \omega_{3}\right)$, while the measured ones are $\left(\omega_{-}, \omega_{0}, \omega_{+}\right)$. The tuning procedure consisted, first, in equalizing the frequencies of the coupling cells of the bridge $\left(\omega_{1}=\omega_{3}\right)$ and then varying the central cell frequency $\omega_{0}$ to get a set of measured data. For $\omega_{1}=\omega_{3}$, a linear relation applies between a combination of the sum and of the ratio of the measured frequencies [3]:

$$
\left(\frac{\omega_{0}^{2}}{\omega_{+}^{2}}+\frac{\omega_{0}^{2}}{\omega_{-}^{2}}=\frac{1-k_{2} / 2}{1+k_{2} / 2}+\frac{\omega_{0}^{4}}{\omega_{+}^{2} \cdot \omega_{-}^{2}} \cdot \frac{1+k_{2} / 2}{1-k_{2} / 2-k_{1}^{2} / 2}\right)
$$

By a linear fit to the data in that formula, it was possible to obtain the first and second coupling coefficients, $\mathrm{k}_{1}$ and $\mathrm{k}_{2}$, so the single cell frequencies could be found from the theory of coupled resonant cavities. In this way, one could correctly tune the cells in the bridge couplers, except for the central cell of the central bridge, which remained $6.5 \mathrm{MHz}$ below the nominal value. This caused a small bump in the field level between tanks 2 and 3 (Figure 4).

The waveguide of the RF feeder line has been brazed tangentially to the central bridge coupler and terminated in a short-circuit at $\lambda / 4$ from an elliptical coupling iris; see Figure 2. The dimension of the iris was determined approximately by applying the Slater's tuning curve method to a series of frequency-domain MAFIA simulations [4]. The matching factor $\beta$ measured finally was 1.14 , corresponding to a reflected input power of $1.6 \%$.

The complete module (tanks and bridge couplers) was assembled and measured before the penultimate brazing, and the length of the tuning rods in the accelerating cells (one per half cell) was adjusted to give the correct overall frequency and the required field flatness. Acting in the same way on the coupling cells, the stop band was adjusted to be $+150 \mathrm{kHz}$. All the rod tuners in a tank, except in the accelerating cells at the ends, are cut to the same length. They were used essentially to compensate the differences in tank frequencies and to act on the stop band, but not to equalize the single cell frequencies. The frequency and field distribution measured before and after the vertical brazing remained the same, as verified by bead-pulling measurements; see Figure 4.

To set the LIBO accelerating field correctly during the power tests, one had to know the cavity shunt impedance R. This was determined by perturbation measurements combined with Superfish computations, yielding $\mathrm{R}=47 \mathrm{M} \Omega$. The dielectric constant of the perturbative element, a nylon wire, was measured beforehand in a test cavity.

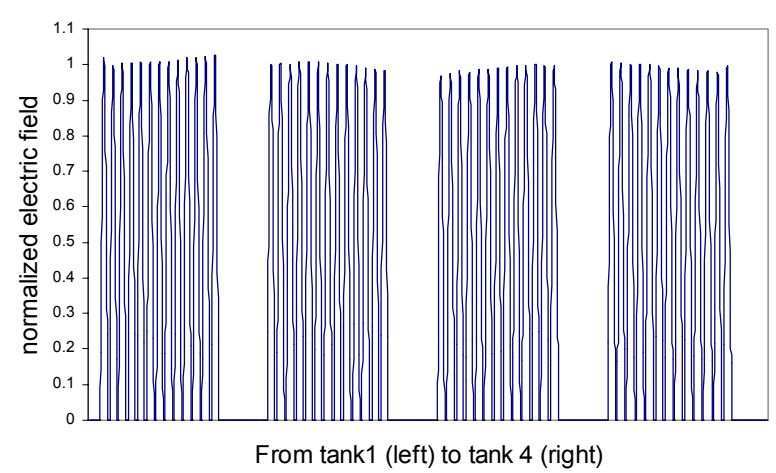

Figure 4: The accelerating field distribution in the four tanks of LIBO shows a uniformity of around 3\%.

\section{CONCLUSION AND OUTLOOK}

The short conditioning time and the very limited number of electrical breakdowns was in our opinion due to the high surface cleanliness of the structure and the thorough and accurate brazing operation. The careful design of the cells and the applied RF tuning procedures contributed also to this success. In the summer 2001, beam acceleration tests will be made at the INFN Laboratory in Catania, Italy, which possesses a cyclotron with the required energy.

The high gradients reached will allow us to reconsider the design of LIBO, possibly using fewer modules for the same final energy. An improved design should then be tested on a second module, made to demonstrate possible industrialization of the project.

\section{ACKNOWLEDGEMENTS}

For the RF power test in LIL, the professional support was provided by the PS-RF group. Mechanical construction and vacuum brazing were done in CERN by dedicated teams led by J.C.Gervais and S.Mathot (both EST). The preparation of the power test, the installation of the LIBO prototype in LIL and the test itself would not have been possible without the spontaneous and efficient help of colleagues from EP, LHC and PS divisions, in particular S. Haider (EP), D. Allard (LHC), G. Rossat (PS), G. Yvon (PS) and M. O'Neil (PS). To all of the above go our deepest thanks.

\section{REFERENCES}

[1] P. Berra et al., "Study, construction and test of a $3 \mathrm{GHZ}$ proton linac-booster (LIBO) for cancer therapy," Proceedings EPAC 2000, Vienna (June 2000), p. 2495.

[2] U. Amaldi et al., "LIBO- A $3 \mathrm{GHZ}$ proton linac booster of $200 \mathrm{MeV}$ for cancer treatment," Proceedings LINAC 98, Chicago (August 1998), p. 633.

[3] D. Davino et al., "Radiofrequency measurements on coupled resonant cavities," to be published.

[4] J. C. Slater, "Microwave Electronics," (D. Van Nostrand, New York, 1950), p. 97. 